\title{
Aged peripheral retinal lesions originating from the ciliary body sweep away the retinal pigmented epithelium
}

\author{
Rana Begum, Glen Jeffery
}

Institute of Ophthalmology, University College London, London, UK

\section{Correspondence to} Professor Glen Jeffery, Institute of Ophthalmology, University College London, 11-43 Bath Street, London EC1V 9EL, UK; g.jeffery@ucl.ac.uk

Accepted 21 February 2012 Published Online First 18 March 2012

\begin{abstract}
Aims To investigate age-related lesions in the far-anterior retina that migrate from the ciliary body (CB) and how they affect the neural retina and retinal pigmented epithelium (RPE).

Methods One eye from three healthy subjects aged 87, 92 and 93 years were used. Retinae were photographed, embedded in resin and then sectioned at $2 \mu \mathrm{m}$.

Results Multiple elliptically shaped lesions were present in the CB. Larger lesions extended into the peripheral retina. Lesions resulted from deposits that had lenticular qualities. These develop centrally along Bruch's membrane sweeping away the RPE, such that piles of RPE cells were present around the deposits that resulted in retinal atrophy. The internal composition of the deposits revealed large numbers of spherical bodies, unlike those seen in drusen. RPE cells adjacent to these deposits and their underlying lesions became highly irregular, with melanin granules spacing themselves out within the cell and adopting similar orientations. This is a highly distinctive feature.

Conclusions These far-anterior deposits were different in nature from drusen in terms of morphology, composition and origin. They swept away the RPE, exposing the Bruch's membrane and isolating the retina, leading to atrophy. They appeared to originate from the $\mathrm{CB}$ and progressed centrally. The deposits may have developed from the ciliary muscle, which would account for their elongated orientation. Their impact on melanin distribution in RPE cells was unexpected and unusual, implying that they release a signal that influences melanin organisation.
\end{abstract}

\section{INTRODUCTION}

In the ageing retina, there is general progressive cell loss, increased extracellular deposition and reduced function. ${ }^{1}$ The progressive deposition of extracellular material has been studied in detail in the human central retina because of the impact of these deposits on visual function. Generally, with age, there is an increase in extracellular deposits and accumulation of drusen. ${ }^{2}{ }^{3}$ In some cases, the central drusen becomes confluent, separating regions of the neural retina from their choroidal blood supply, which results in geographic atrophy and age-related macular degeneration. ${ }^{4}$

Retinal deposits are not uniform. Central human deposits can be large and confluent, while those found in equatorial regions tend to be more solid and have distinct fluorescent properties and morphological features. Equatorial deposits are also much smaller than those in the centre and are relatively symmetric. ${ }^{5} 6$ While studies have examined both central and equatorial drusen, few have examined the obvious and distinct deposits and lesions that commonly appear and progress in the far-anterior retina, as these have little direct impact on vision. Because of their clear spherical appearance, these deposits have been called cystoids. ${ }^{7-9}$ Their origins reside in the ciliary epithelium; however, they extend and develop into the retina. ${ }^{10}$ Here, we examine these deposits and determine whether they share features with those located more centrally or whether they have a totally distinct form. We also address their impact on the retinal pigmented epithelium (RPE).

\section{METHODS}

Aged formalin fixed eyes, from the eye bank at Moorfields Eye Hospital, were opened and examined at the macroscopic level for the presence of peripheral lesions. These eyes were considered healthy, as they did not have any obvious signs of cataract surgery or other significant age-related changes. One eye from three subjects aged 87, 92 and 93 years with clear far-anterior lesions was selected (figure 1A). These were photographed under normal bright field and fluorescence. The fluorescent stimulus was $480 \mathrm{~nm}$, similar to that used to identify equatorial drusen. ${ }^{5} 6$ The lesioned area including normal tissue around it was dissected; this tissue extended beyond the retina and into the ciliary body (CB). The sizes of the dissected regions varied with lesion size. Photoshop was used on images from whole-mounted tissue to measure the $\mathrm{x}$ - and $\mathrm{y}$-axis of 10 deposits/lesions to describe their shape. The lesions had an average length of $58 \mu \mathrm{m}$ whereas the average width was $20 \mu \mathrm{m}$. Tissues from similar eccentricities that appeared macroscopically normal were also dissected free and used as control regions.

Retinal regions that were dissected free were placed between two microscope slides to keep them flat. All retinal samples were then dehydrated using a graded series of alcohols. The samples were then placed on tissue paper to remove surplus liquid, infiltrated with Historesin (Kulzer Technovi 7100, $\mathrm{TAAB}$, Alermaston, UK) overnight and the resin was polymerised the following morning. The tissues were sectioned at $2 \mu \mathrm{m}$, mounted onto slides, dried before staining with toluidine blue and covered with a cover slip.

\section{RESULTS \\ Whole-mount preparations}

The flat mounted tissue from the far-anterior retina was commonly distorted with extensive deposits of 
Figure 1 Peripheral retinal flat mount with multiple lesions. (A) Circled regions show small (far right), intermediate sized (far left) and advance (centre) lesions. Small lesions are confined to the ciliary body (CB) and appear as small individual units. This is apparent in B. Larger, more advanced deposits appear to consist of smaller deposits/lesions merged together, forming heterogeneous structures, that span the ciliary border and progress into the retina. (B) Smaller lesions appear as elliptical shapes, pointing centrally towards the optic nerve head, with the long axis to short axis in a 3:1 ratio. Scale bar $A=3 \mathrm{~mm}$, $\mathrm{B}=30 \mu \mathrm{m}$. This figure is produced in colour in the online journal-please visit the website to view the colour figure.
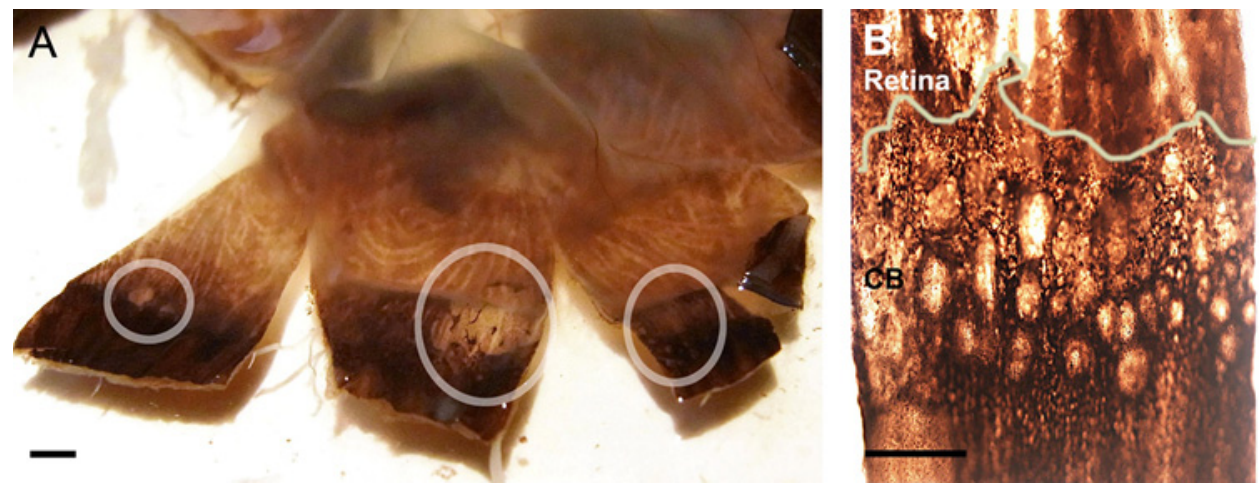

varying sizes. The smaller deposits/lesions were confined to the $\mathrm{CB}$ bordering the retina. Those that were larger crossed this border and extended to varying distances into the retina. Figure 1 shows both large lesions (figure $1 \mathrm{~A}$ ) and a series of much smaller lesions (figure 1B). While the larger lesions appeared to be derived from multiple smaller lesions looking like a series of fused circles, the smaller lesions were not circular but elliptical with their long axis always aligned towards the central retina. When the long axis of the smaller lesions was measured against the short axis, the resulting ratio was approximately $3: 1$ consistently.

In whole mounts, prior to dehydration, the areas affected by the lesions showed a series of distinct features irrespective of the size. First, when the retina was removed, the lesions were translucent and lenticular in nature, and most of the underlying RPE had been removed by the deposit. It was clear that the lesioned areas were occupied by translucent bodies whose plane of focus was above that of the RPE layer. In larger lesions, these bodies had clear boundaries (figure 2A). Equatorial drusen have distinct fluorescent properties when viewed with stimulating light at a wavelength of around $480 \mathrm{~nm} .{ }^{5}$ The deposits occupying the lesioned area also had a green fluorescence at this wavelength (figure $2 \mathrm{~B}, \mathrm{C}$ ) but this did not distinguish them from the surrounding tissue. The three images in the upper panel in figure 2 were all taken at slightly different focal planes, as can be seen by the clarity of the RPE at the edge of the deposit/lesion. Similar pictures of smaller deposits are shown in figure $2 \mathrm{D}, \mathrm{E}$. These two pictures show the RPE around the two small deposits. Figure 2D focuses on the RPE morphology surrounding the deposit, whereas figure $2 \mathrm{E}$ shows the RPE beneath the deposit. Again, the two images are in a different focal plane, revealing the clear lenticular nature of the deposits.

The RPE cells under every deposit/lesion were disrupted and their number was markedly reduced. The reason for this is apparent in figure 2F. The larger deposits have an accumulation of RPE cells on the edge that faces the central retina. This gives the impression that the deposit develops towards the centre of the retina and in doing so sweeps the RPE cells clear of the Bruch's membrane (BM). All larger deposits displayed this feature, which can also be seen at the macroscopic level (figure 1A). The only exception to this were rows of RPE cells trapped in larger deposits, which were interpreted as being the product of entrapment by deposit fusion.

\section{Sectioned preparations}

When the deposits/lesions were viewed in section, there was further evidence that the translucent deposits (figure 2G, asterisk) were removing the RPE; some of these cells were swept above the leading edge of the deposit where they accumulated. The retina below was largely degenerate. The internal organisation of the deposit revealed a large number of spherical bodies within a heterogeneous structure.

The tissue adjacent to the deposits showed progressive changes depending on proximity. Approximately $30 \mu \mathrm{m}$ away from the deposit, the structure of the retina could be identified (figure 3A). However, on moving towards the deposit, fewer RPE cells were found on the BM and although some photoreceptors were still present, the retinal structure was largely lost (figure 3B). Directly adjacent to the deposit, all of the RPE was missing and the overall structure of the retina was lost (figure $3 \mathrm{C}$ ).

There were very marked changes in the organisation of the RPE cells around the deposits, which can be partially viewed in figure 3A. Away from the deposit/lesion, photoreceptor topography was relatively normal for an aged retina, with most pigments in the RPE located apically within the cells (figure 4A). However, when the RPE was close to a deposit/lesion, there was a marked change in the topography (figure $4 \mathrm{~B}$ ). Here the RPE cells started to round up, perhaps due to the loss of their junctional connections. But, more obviously, the melanin distribution in the cells changed. Melanin granules became relatively evenly distributed throughout the cell and their numbers appeared to increase. Furthermore, all the melanin granules adopted the same orientation. Melanin granules are commonly coin shaped and hence can appear either round-similar to looking at a coin face on-or highly elliptical-similar to viewing a coin end on. All the melanin granules close to the deposits/lesions were round. Hence, these structures had changed their distribution, becoming relatively evenly distributed and their orientation was unified.

\section{DISCUSSION}

With age, the neuronal population of the retina is progressively erroded. ${ }^{11} 12$ There is also a progressive increase in inflammation and deposition of extracellular material. ${ }^{2}{ }^{3}$ The origin of this material is unclear but, ultimately, it may due to failure of the ageing RPE to appropriately digest the photoreceptor outer segments. These deposits accumulate on the outer segments and 
Figure 2 Deposits in whole mount and sectioned preparations. (A) Low magnification image of lesioned area from figure 1; bright field microscopy shows a large deposit/lesion with a clear boundary. $(B, C)$ Stimulation at $480 \mathrm{~nm}$. The deposits had similar fluorescent properties as equatorial drusen but this did not distinguish them from the background. All three images in $\mathrm{A}-\mathrm{C}$ were in a different focal plane. (D) Images of two smaller lesions. Here the surrounding retinal pigmented epithelium (RPE) cells were in focus, whereas those beneath the lesions were not; the opposite was observed in $E$, suggesting that the deposit forming lesions had a lens like property. (F) RPE cells lying under the deposit were affected; these cells begin to accumulate at the edge and appear to be pushed back from the Bruch's membrane (BM) towards the central retina. $(G)$ Deposits $\left({ }^{*}\right)$ develop along the BM and start to remove the RPE. The internal structure of the deposits reveals multiple spherical bodies and fibrous elements. Scale bar $A-C=15 \mu \mathrm{m}, D$ and $E=30 \mu \mathrm{m}$, $\mathrm{F}=20 \mu \mathrm{m}, \mathrm{G}=50 \mu \mathrm{m}$. This figure is produced in colour in the online journal-please visit the website to view the colour figure.
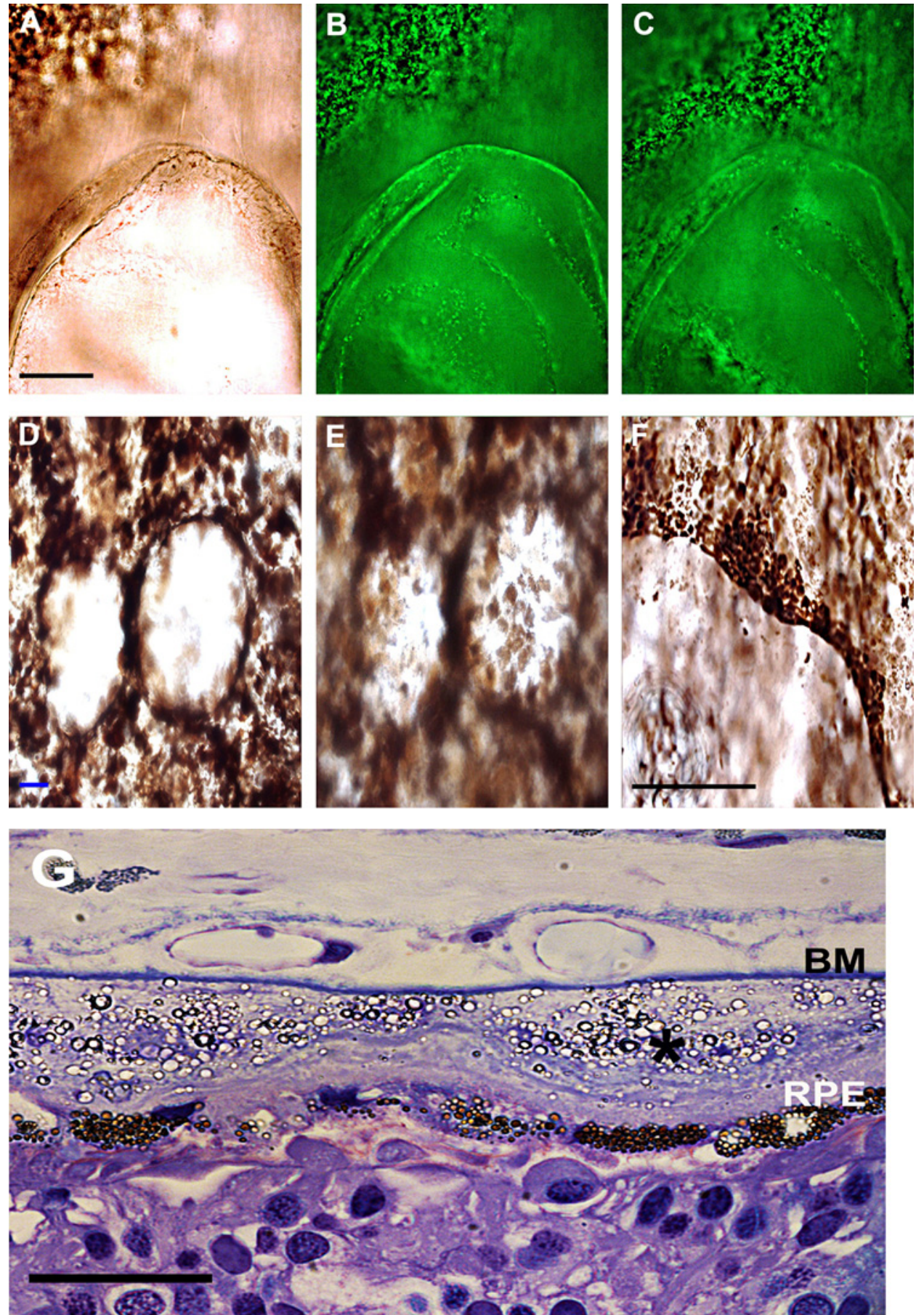

on the BM. ${ }^{3}$ In both cases, they contain neurotoxic amyloid and, in the case of those on the BM, they progressively restrict metabolic flow. ${ }^{13}$ The deposits that accumulate on the BM differ

depending on the location and some probably lead to drusen formation. Central drusen can be large and confluent and appear opaque in fundus photographs. ${ }^{6}$ Those in equatorial regions are

Figure 3 Progressive changes in the retinal structure associated with deposits. As the deposits progressed through the retina, the relatively normal (A) retinal architecture began to alter. (B) Closer to the deposit, there was an initial loss of photoreceptors $(\mathrm{Ph})$ and retinal pigmented epithelium (RPE) cells. (C) Complete loss of structure was found adjacent to the deposit. Scale bar $A=15 \mu \mathrm{m}$. This figure is produced in colour in the online journal-please visit the website to view the colour figure.
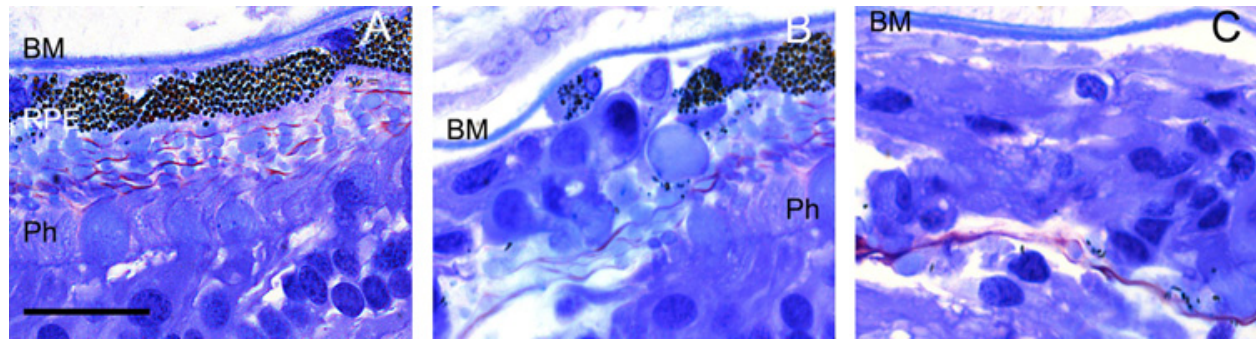
Figure 4 Distribution and orientation of melanin in retinal pigmented epithelium (RPE) cells. (A) Normal, deposit-free regions showed a relatively normal aged far-peripheral retina, with photoreceptors (Ph), Bruch's membrane (BM) and the RPE cells. The RPE cells were tightly packed; more importantly, their pigment was apically located, which is the predisposition of normal RPE melanin. (B) Closer to the deposits, the RPE cells showed increased pigmentation and cells began to round up probably due to the loss of their junctional connections. Unexpectedly, the melanin granules became evenly distributed, filling the cell and all adopting the same orientation. Scale bar $A=5 \mu \mathrm{m}, B=7 \mu \mathrm{m}$. This figure is produced in colour in the online journal-please visit the website to view the colour figure.

more focal, hard and smaller, with different fluorescent signatures. ${ }^{5}$ This implies that deposits at the two locations have different biochemical characteristics. However, neither has the characteristics found in the peripheral deposits described in this study, which were clear and lenticular both before and after dehydration. Internally, they appeared to contain spherical bodies; they also seemed to be firmly attached to the BM, as they generally swept away the RPE isolating the retina.

These deposits maybe related to the lens zonules radiating towards the ciliary muscles. This would explain not only their geographic origin, but also the marked difference in their physical characteristics, being unlike the drusen that are found more centrally; however, this remains to be demonstrated. While ciliary muscles remain contractile with age, a number of changes take place including reductions in unaccommodated muscle diameter ${ }^{14}$ and a watershed of changes that occur over the age of 70 years, which include decreased cross-sectional area, increased connective tissue content and reduced nuclei numbers. ${ }^{15}$ Deposits in the CB have been noted before; in some cases they can appear as small white nodules called Fuch's adenoma, which can be associated with cataracts; ${ }^{16}$ however, these do not fit the description of the material described in this study. Furthermore, the existence of lipid-rich deposits at this location has also been noted previously, although their impact on the retina, their internal structure and physical characteristics were not described. ${ }^{9}$ Blessig $^{7}$ and Iwanoff $^{8}$ were the first to establish the term 'cystoid degeneration' which describes the accumulation of transparent materials within the retina, but the impact of these on adjacent tissue was largely left unaddressed.

One of the key reasons why the lesions described here have received so little attention is that it is thought they have little direct impact on vision. However, two specific features of the faranterior retina are affected by the progressive development of these deposits and the subsequent loss of retinal function at extreme eccentricities. First, the human far-anterior retina is cone dominated. ${ }^{17-19}$ The reason for this is unclear but probably relates to the fact that during development, at any retinal location, cones are generated before rods. ${ }^{20}$ Hence termination of cell production in the peripheral retina will result in a relatively cone-rich region. These cones tend to be large $e^{17-19}$ and are almost certainly only subject to diffuse illumination, but it is possible that they have a functional role that does not relate to spatial vision.

Second, this region also contains a plexus of melanopsin containing cells and their processes that course around the retinal rim. ${ }^{21}$ Melanopsin containing cells are heterogeneous. ${ }^{22} 23$ In addition, it has been argued that some may mediate crude pattern vision. ${ }^{24}$ But cells located at the retinal rim will be unable to do this because they are located at the extremities; like the cones, they will not receive spatial information, only diffuse illumination. Consequently, their primary role must be that originally ascribed to the melanopsin system - that of circadian regulation. ${ }^{25}$ Many of these peripheral melanopsin cells may be lost because of the progression of the deposits described here and their loss may contribute to reduced circadian behaviour in older humans. ${ }^{25}$

Acknowledgements The authors would like to thank Jaimie Hoh Kam and Vivian Lee for their time and help.

Contributors RB undertook the majority of the lab work and the manuscript was written jointly by both authors.

Funding This study was funded by the Rosetrees Trust.

Competing interests None.

Patient consent Obtained.

Ethics approval This study was approved by the Human Tissue Authority and was conducted in accordance with the guidelines set by University College London.

Provenance and peer review Not commissioned; externally peer reviewed.

\section{REFERENCES}

1. Gao HH, Hollyfield JG. Aging of the human retina. Differential loss of neurons and retinal pigmented epithelial cells. Invest Ophthalmol Vis Sci 1992; 33:1-17.

2. Anderson DH, Mullins RF, Hageman GS, et al. A role for local inflammation in the formation of drusen in the aging eye. Am J Ophthalmol 2002:134:411-31.

3. Hoh Kam J, Lenassi E, Jeffery G. Viewing ageing eyes: diverse sites of amyloid Beta accumulation in the ageing mouse retina and the up-regulation of macrophages. PLoS One 2010:10:e13127.

4. Cook HL, Patel PJ, Tufail A. Age-related macular degeneration: diagnosis and management. Br Med Bull 2008:85:127-49.

5. Lengyel I, Tufail A, Hosaini HA, et al. Association of drusen deposition with choroidal intercapillary pillars in the aging human eye. Invest Ophthalmol Vis Sci 2004:45:2886-92.

6. Hageman GS, Gehrs K, Johnson LV, et al. Age-related macular degeneration (AMD) In: Kolb H, Fernandez E, Nelson R, eds. The Organization of the Retina and Visual System. Salt Lake City (UT): University of Utah Health Sciences Center, 1995.

7. Blessig R. De retininae textura disquisitiones microscopicae. Doctoral thesis. Tartu 1855.

8. Iwanoff A. Bietrage zue Normalen und Pathologischen Anatomie des Augus-das Oedem der Netzhaut. Arch Ophthalmol 1869;15:88-105.

9. O'Malley PF, Allen RA. Peripheral cystoid degeneration of the retina. Arch Ophthalmol 1967:769-76.

10. Rutnin U, Schepens CL. Fundus appearance in normal eyes. 3. Peripheral degenerations. Am J Opthalmol 1967;64:1040-62.

11. Curcio CA, Millican CL, Allen KA, et al. Aging of the human photoreceptor mosaic: evidence for selective vulnerability of rods in central retina. Invest Ophthalmol Vis Sci 1993:34:3278-96.

12. Cunea A, Jeffery G. The ageing photoreceptor. Vis Neurosci 2007;24:151-5 
13. Yoshida T, Ohno-Matsui $\mathrm{K}$, Ichinose $\mathrm{S}$, et al. The potential role of amyloid beta in the pathogenesis of age-related macular degeneration. J Clin Invest

2005;115:2793-800.

14. Strenk SA, Semmlow JL, Strenk LM, et al. Age-related changes in human ciliary muscle and lens: a magnetic resonance imaging study. Invest Ophthalmol Vis Sci 1999;40:1162-9.

15. Nishida SS, Mizutani SS. Quantitative and morphometric studies of age-related changes in human ciliary muscle. Jpn J Ophthalmol 1992;36:380-7.

16. Klintworth GK. Fuchs Adenoma. The Eye Pathologist, 2002. http://eyepathologist com/disease.asp?IDNUM=304980 (accessed 11 Jan 2011).

17. Greeff R. Mikroscopische Anatomie der Sehnerven und der Netzhaut. In: von Graefe A, Saemisch T, eds. Handbuch der gesamten Augenheilkunde. Berlin: Springer, 1900.

18. Williams R. The human retina has a cone-enriched rim. Vis Neurosci 1991;6:403-6
19. Mollon JD, Regan BC, Bowmaker JK. What is the function of the cone-rich rim of the retina? Eye (Lond) 1998;12:548-52.

20. La Vail MM, Rapaport DH, Rakic P. Cytogenesis in the monkey retina. J Comp Neurol 1991;309:86-114.

21. Vugler AA, Semo M, Joseph A, et al. Survival and remodeling of melanopsin cells during retinal dystrophy. Vis Neurosci 2008;25:125-38.

22. Chen SK, Badea TC, Hattar S. Photoentrainment and pupillary light reflex are mediated by distinct populations of ipRGCs. Nature 2011;476:92-5.

23. Schmidt TM, Do MT, Dacey D et al. Melanopsin-positive Intrinsically photosensitive retinal ganglion cells: from form to function. J Neurosci 2011;45:16094-101.

24. McNeill DS, Sheely CJ, Ecker JL, et al. Development of melanopsin-based irradiance detecting circuitry. Neural Dev 2011:6:8.

25. Paul KN, Saafir TB, Tosi G. The role of retinal photoreceptors in the regulation of circadian rhythms. Rev Endocr Metab Disord 2009:10:271-8. 